\title{
Coarse Differentiation and Multi-flows in Planar Graphs
}

\author{
James R. Lee · Prasad Raghavendra
}

Received: 6 May 2008 / Revised: 4 April 2009 / Accepted: 7 April 2009 / Published online: 22 April 2009 (C) Springer Science+Business Media, LLC 2009

\begin{abstract}
We show that the multi-commodity max-flow/min-cut gap for seriesparallel graphs can be as bad as 2, matching a recent upper bound (Chakrabarti et al. in 49th Annual Symposium on Foundations of Computer Science, pp. 761-770, 2008) for this class, and resolving one side of a conjecture of Gupta, Newman, Rabinovich, and Sinclair.

This also improves the largest known gap for planar graphs from $\frac{3}{2}$ to 2 , yielding the first lower bound that does not follow from elementary calculations. Our approach uses the coarse differentiation method of Eskin, Fisher, and Whyte in order to lower bound the distortion for embedding a particular family of shortest-path metrics into $L_{1}$.
\end{abstract}

Keywords Discrete metric spaces $\cdot$ Embeddings $\cdot$ Sparsest cut $\cdot$ Metric differentiation

\section{Introduction}

Low-distortion metric embeddings have become an increasingly powerful tool in studying the relationship between cuts and multi-commodity flows in graphs. For background on the field of metric embeddings and their applications in theoretical computer science, we refer to Matoušek's book [26, Chap. 15], the surveys [19, 23], and the compendium of open problems [25].

One of the central connections lies in the correspondence between low-distortion $L_{1}$ embeddings, on the one hand, and the Sparsest Cut problem (see, e.g., [2-4,

J.R. Lee's research partially supported by NSF CAREER award CCF-0644037. Part of this research was completed while the author was a postdoctoral fellow at the Institute for Advanced Study, Princeton.

P. Raghavendra's research supported in part by NSF grant CCF-0343672.

J.R. Lee $(\bowtie) \cdot$ P. Raghavendra

University of Washington, Seattle, USA

e-mail: jrl@cs.washington.edu 
24]) and concurrent multi-commodity flows (see, e.g., $[12,17])$ on the other. This relationship allows one to bring sophisticated geometric and analytic techniques to bear on classical problems in graph partitioning and in the theory of network flows. In the present paper, we show how techniques developed initially in geometric group theory can be used to shed new light on the connections between sparse cuts and multi-commodity flows in planar graphs.

Multi-commodity Flows and Sparse Cuts Let $G=(V, E)$ be an undirected graph, with a capacity $C(e) \geq 0$ associated to every edge $e \in E$. Assume that we are given $k$ pairs of vertices $\left(s_{1}, t_{1}\right), \ldots,\left(s_{k}, t_{k}\right) \in V \times V$ and $D_{1}, \ldots, D_{k} \geq 1$. We think of the $s_{i}$ as sources, the $t_{i}$ as targets, and the value $D_{i}$ as the demand of the terminal pair $\left(s_{i}, t_{i}\right)$ for commodity $i$.

In the Concurrent MaxFlow problem the objective is to maximize the fraction $\lambda$ of the demand that can be shipped simultaneously for all the commodities, subject to the capacity constraints. Denote this maximum by $\lambda^{*}$. A straightforward upper bound on $\lambda^{*}$ is the sparsest cut ratio $\Phi^{*}$ defined as follows. Given any subset $S \subseteq V$, we write

$$
\Phi(S)=\frac{\sum_{u v \in E} C(u v) \cdot\left|\mathbf{1}_{S}(u)-\mathbf{1}_{S}(v)\right|}{\sum_{i=1}^{k} D_{i} \cdot\left|\mathbf{1}_{S}\left(s_{i}\right)-\mathbf{1}_{S}\left(t_{i}\right)\right|},
$$

where $\mathbf{1}_{S}$ is the characteristic function of $S$. The value $\Phi^{*}=\min _{S \subseteq V} \Phi(S)$ is the minimum over all cuts (partitions) of $V$, of the ratio between the total capacity crossing the cut and the total demand crossing the cut. In the case of a single commodity (i.e., $k=1$ ) the classical MaxFlow-MinCut theorem states that $\lambda^{*}=\Phi^{*}$, but in general this is no longer the case. It is known $[4,24]$ that $\Phi^{*}=O(\log k) \lambda^{*}$. This result is perhaps the first striking application of metric embeddings in combinatorial optimization (specifically, it uses Bourgain's embedding theorem [6]).

Indeed, the connection between $L_{1}$ embeddings and multi-commodity flow/cut gaps can be made quite precise. For a graph $G$, let $c_{1}(G)$ represent the largest distortion necessary to embed any shortest-path metric on $G$ into $L_{1}$ (i.e., the maximum over all possible assignments of nonnegative lengths to the edges of $G$ ). Then $c_{1}(G)$ gives an upper bound on the ratio between the sparsest cut ratio and the maximum flow for any multi-commodity flow instance on $G$ (i.e., with any choices of capacities and demands) [4, 24]. Furthermore, this connection is tight in the sense that there is always a multi-commodity flow instance on $G$ that achieves a gap of $c_{1}(G)$ [17].

Despite significant progress $[7,8,12,17,28]$, some fundamental questions are still left unanswered. As a prime example, consider the well-known planar embedding conjecture $[17,19,23,25]$ :

There exists a constant $C$ such that every planar graph metric embeds into $L_{1}$ with distortion at most $C$.

In initiating a systematic study of $L_{1}$ embeddings [17] for minor-closed families, Gupta, Newman, Rabinovich, and Sinclair put forth the following vast generalization of this conjecture (we refer to [14] for the relevant graph theory).

Conjecture 1 (Minor-closed Embedding Conjecture) If $\mathcal{F}$ is any nontrivial minorclosed family, then $\sup _{G \in \mathcal{F}} c_{1}(G)<\infty$. 
Lower Bounds on the Multi-commodity Max-flow/Min-cut Ratio in Planar Graphs While techniques for proving upper bounds on the $L_{1}$-distortion required to embed such families have steadily improved, progress on lower bounds has been significantly slower, and recent breakthroughs in lower bounds for $L_{1}$ embeddings of discrete metric spaces that rely on discrete Fourier analysis [20, 21] do not apply to excluded-minor families.

The best previous lower bound on $c_{1}(G)$ when $G$ is a planar graph occurred for $G=K_{2, n}$, i.e., the complete $2 \times n$ bipartite graph. By a straightforward generalization of the lower bound of Okamura and Seymour [28], it is possible to show that $c_{1}\left(K_{2, n}\right) \rightarrow \frac{3}{2}$ as $n \rightarrow \infty$ (see also [1] for a simple proof of this fact in the dual setting).

We show that, in fact, there is an infinite family of series-parallel (and hence, planar) graphs $\left\{G_{n}\right\}$ such that $\lim _{n \rightarrow \infty} c_{1}\left(G_{n}\right)=2$; this is not only a new lower bound for planar graphs but yields an optimal lower bound on the $L_{1}$-distortion (and hence, the flow/cut gap) for series-parallel graphs. The matching upper bound was recently proved in [8].

\subsection{Results and Techniques}

Our lower bound approach is based on exhibiting local rigidity for pieces of metric spaces under low-distortion embeddings into $L_{1}$ (which we take to mean $L_{1}([0,1]$ ) throughout). This circle of ideas and the relationship to theory of metric differentiation are a long-studied phenomena in geometric analysis (see, e.g., [5, 9, 18, 29]). More recently, they have been applied to the study of $L_{1}$ embeddings [10, 11] based on local rigidity results for sets of finite perimeter in the Heisenberg group [16]; see [22] for the relevance to integrability gaps for the Sparsest Cut problem.

The philosophy underlying these approaches is as follows. Suppose that $X$ is the shortest-path metric on some graph. As is well known, an embedding of $X$ into $L_{1}$ induces a distribution on cuts in $X$, i.e., subsets $S \subseteq X$ (see Sect. 2.2). If the embedding has low distortion, then in a precise quantitative sense, most of the weight of the cuts in this distribution is concentrated on subsets $S$ with small perimeter, i.e., which cut few edges of the graph. For us, rigidity then refers to the phenomenon (a property of $X$ ) that these cuts of small perimeter must have some canonical form. Local rigidity refers to the fact that this conclusion is a local one-we can only conclude that for some small ball $B$ in $X$, "most" of the cuts $S \cap B$ are close to canonical. Finally, we use this to prove lower bounds by showing that any $L_{1}$ embedding composed primarily of canonical cuts cannot have small distortion.

Our basic approach is simple; we know that $c_{1}\left(K_{2, n}\right) \leq \frac{3}{2}$ for every $n \geq 1$. Recall that any $L_{1}$ embedding can be expressed as a convex combination of cuts. Suppose it were possible to restrict the kind of cuts that appear in a $L_{1}$ embedding, then presumably the distortion would be greater than $\frac{3}{2}$. Specifically, consider $s, t \in V\left(K_{2, n}\right)$ which constitute the partition of size 2 . Say that a cut $S \subseteq V\left(K_{2, n}\right)$ is monotone with respect to $s$ and $t$ if every simple $s-t$ path in $K_{2, n}$ has at most one edge crossing the cut $(S, \bar{S})$. It is not difficult to show that if an $L_{1}$ embedding is composed entirely of cuts which are monotone with respect to $s$ and $t$, then that embedding must have distortion at least $2-\frac{2}{n}$. 
Consider now the recursively defined family of graphs $K_{2, n}^{\oslash k}$, where $K_{2, n}^{\oslash 1}=K_{2, n}$, and $K_{2, n}^{\oslash k}$ arises by replacing every edge of $K_{2, n}^{\oslash k-1}$ with a copy of $K_{2, n}$. More precisely, the graph $K_{2, n}$ can be thought of as $n$ disjoint paths of length 2 between two vertices $s, t$ which constitute the partition of size 2 . Replace each edge $(u, v)$ in $K_{2, n}^{\oslash k-1}$ with a copy of $K_{2, n}$ by identifying the endpoints $\{u, v\}$ with the vertices $\{s, t\}$ in the copy of $K_{2, n}$. The family $\left\{K_{2,2}^{\oslash k}\right\}_{k \geq 1}$ is the well-known diamond graphs of [17, 27]. We show that in any low-distortion embedding of $K_{2, n}^{\oslash k}$ into $L_{1}$, for $k \geq 1$ large enough, it is possible to find a (metric) copy of $K_{2, n}$ for which the induced embedding is composed almost entirely of monotone cuts. The claimed distortion bound follows, i.e., $\lim _{n, k \rightarrow \infty} c_{1}\left(K_{2, n}^{\oslash k}\right)=2$. In Sect. 5, we exhibit embeddings which show that for every fixed $n, \lim _{k \rightarrow \infty} c_{1}\left(K_{2, n}^{\oslash k}\right)<2$, thus it is necessary to have the base graphs grow in size.

The ability to find these monotone copies of $K_{2, n}$ inside a low-distortion $L_{1}$ embedding of $K_{2, n}^{\oslash k}$ arises from two sources. The first is the coarse differentiation technique of Eskin, Fisher, and Whyte [15], which gives a discrete approach to finding local regularity in distorted paths; this is carried out in Sect. 3. The second aspect is the relationship between regularity and monotonicity for $L_{1}$ embeddings which is expounded upon in Sect. 3.2 and relies on the well-known fact that every $L_{1}$ embedding decomposes in a certain way into a distribution over cuts.

\section{Preliminaries}

For a graph $G$, we will use $V(G), E(G)$ to denote the sets of vertices and edges of $G$, respectively. Sometimes we will equip $G$ with a nonnegative length function len $: E(G) \rightarrow \mathbb{R}_{+}$, and we let $d_{\text {len }}$ denote the shortest-path (semi-)metric on $G$. We say that len is a reduced length if $d_{\operatorname{len}}(u, v)=\operatorname{len}(u, v)$ for every $(u, v) \in E(G)$. All length functions considered in the present paper will be reduced. We will write $d_{G}$ for the path metric on $G$ if the length function is implicit. For an integer $n$, let $K_{2, n}$ denote the complete bipartite graph with 2 vertices on one side, and $n$ on the other. We will use the notation $\left\{x_{i}\right\}_{i=1}^{k}$ to denote a ordered sequence of $k$ elements. All sequences considered in this work are ordered unless otherwise specified.

\section{$2.1 s-t$ Graphs and $\oslash$-products}

An $s-t$ graph $G$ is a graph which has two distinguished vertices $s, t \in V(G)$. For an $s-t$ graph, we use $s(G)$ and $t(G)$ to denote the vertices labeled $s$ and $t$, respectively. Throughout this article, the graphs $K_{2, n}$ are considered $s-t$ graphs in the natural way (the two vertices forming one side of the partition are labeled $s$ and $t$ ).

Definition 2.1 (Composition of $s-t$ Graphs) Given two $s-t$ graphs $H$ and $G$, define $H \oslash G$ to be the $s-t$ graph obtained by replacing each edge $(u, v) \in E(H)$ by a copy of $G$ (see Fig. 1). Formally,

- $V(H \oslash G)=V(H) \cup(E(H) \times(V(G) \backslash\{s(G), t(G)\}))$. 

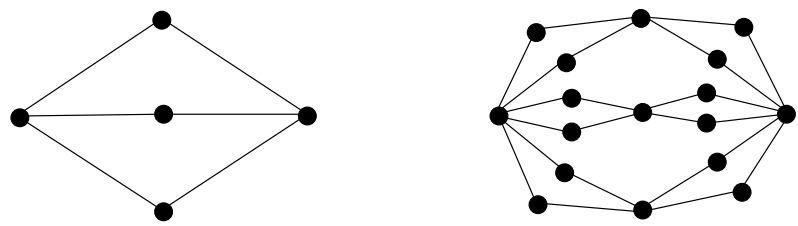

Fig. 1 A single edge $H, H \oslash K_{2,3}$, and $H \oslash K_{2,3} \oslash K_{2,2}$

- For every edge $e=(u, v) \in E(H)$, there are $|E(G)|$ edges,

$$
\begin{aligned}
& \left\{\left(\left(e, v_{1}\right),\left(e, v_{2}\right)\right) \mid\left(v_{1}, v_{2}\right) \in E(G) \text { and } v_{1}, v_{2} \notin\{s(G), t(G)\}\right\} \\
& \quad \cup\{(u,(e, w)) \mid(s(G), w) \in E(G)\} \cup\{((e, w), v) \mid(w, t(G)) \in E(G)\} .
\end{aligned}
$$

- $s(H \oslash G)=s(H)$ and $t(H \oslash G)=t(H)$.

If $H$ and $G$ are equipped with length functions $\operatorname{len}_{H}$ and $\operatorname{len}_{G}$, respectively, we define len $H \oslash G$ as follows. Using the preceding notation, for every edge $e=(u, v) \in$ $E(H)$,

$$
\begin{aligned}
\operatorname{len}\left(\left(e, v_{1}\right),\left(e, v_{2}\right)\right) & =\frac{\operatorname{len}_{H}(e)}{d_{\operatorname{len}_{G}}(s(G), t(G))} \operatorname{len}_{G}\left(v_{1}, v_{2}\right) \\
\operatorname{len}(u,(e, w)) & =\frac{\operatorname{len}_{H}(e)}{d_{\operatorname{len}_{G}}(s(G), t(G))} \operatorname{len}_{G}(s(G), w) \\
\operatorname{len}((e, w), v) & =\frac{\operatorname{len}_{H}(e)}{d_{\operatorname{len}_{G}(s(G), t(G))} \operatorname{len}_{G}(w, t(G))}
\end{aligned}
$$

This choice implies that $H \oslash G$ contains an isometric copy of $\left(V(H), d_{\operatorname{len}_{H}}\right)$.

Observe that there is some ambiguity in the definition above, as there are two ways to substitute an edge of $H$ with a copy of $G$; thus we assume that there exists some arbitrary orientation of the edges of $H$. However, for our purposes, the graph $G$ will be symmetric, and thus the orientations are irrelevant.

Definition 2.2 (Recursive Composition) For an $s-t$ graph $G$ and a number $k \in \mathbb{N}$, we define $G^{\oslash k}$ inductively by letting $G^{\oslash 0}$ be a single edge of unit length and setting $G^{\oslash k}=G^{\oslash k-1} \oslash G$.

The following result is straightforward.

Lemma 2.3 (Associativity of $\oslash$ ) For any three graphs $G_{1}, G_{2}, G_{3}$, we have $\left(G_{1} \oslash\right.$ $\left.G_{2}\right) \oslash G_{3}=G_{1} \oslash\left(G_{2} \oslash G_{3}\right)$, both graph-theoretically and as metric spaces.

Definition 2.4 For two graphs $G, H$, a subset of vertices $X \subseteq V(H)$ is said to be a copy of $G$ if there exists a bijection $f: V(G) \rightarrow X$ with distortion 1, i.e., $d_{H}(f(u), f(v))=c \cdot d_{G}(u, v)$ for some scaling factor $c>0$. 
Now we make the following two simple observations about copies of $H$ and $G$ in $H \oslash G$.

Observation 2.5 The graph $H \oslash G$ contains $|E(H)|$ distinguished copies of the graph $G$, one copy corresponding to each edge in $H$.

Observation 2.6 The subset of vertices $V(H) \subseteq V(H \oslash G)$ form an isometric copy of $H$.

For any graph $G$, we can write $G^{\oslash N}=G^{\oslash k-1} \oslash G \oslash G^{N-k}$. By Observation 2.5, there are $\left|E\left(G^{\oslash k-1}\right)\right|=|E(G)|^{k-1}$ copies of $G$ in $G^{\oslash k-1} \oslash G$. Now using Observation 2.6, we obtain $|E(G)|^{k-1}$ copies of $G$ in $G^{\oslash N}$. We refer to these as the level- $k$ copies of $G$, and their vertices as level-k vertices.

In the case of $K_{2, n}^{\oslash N}$, we will use a compact notation to refer to the copies of $K_{2, n}$. For two level- $k$ vertices $x, y \in V\left(K_{2, n}^{\oslash N}\right)$, we will use $K_{2, n}^{(x, y)}$ to denote the copy of $K_{2, n}$ for which $x$ and $y$ are the $s-t$ points. Note that such a copy does not exist between all pairs of level- $k$ vertices.

\subsection{Cuts and $L_{1}$ Embeddings}

Cuts A cut of a graph is a partition of $V$ into $(S, \bar{S})$ - we sometimes refer to a subset $S \subseteq V$ as a cut as well. A cut gives rise to a semi-metric; using indicator functions, we can write the cut semi-metric as $\rho_{S}(x, y)=\left|\mathbf{1}_{S}(x)-\mathbf{1}_{S}(y)\right|$. A fact central to our proof is that embeddings of finite metric spaces into $L_{1}$ are equivalent to sums of positively weighted cut metrics over that set (for a simple proof of this, see Sect. 4.2 of [13]).

A cut measure on $G$ is a function $\mu: 2^{V} \rightarrow \mathbb{R}_{+}$for which $\mu(S)=\mu(\bar{S})$ for every $S \subseteq V$. Every cut measure gives rise to an embedding $f: V \rightarrow L_{1}$ for which

$$
\|f(u)-f(v)\|_{1}=\int\left|\mathbf{1}_{S}(u)-\mathbf{1}_{S}(v)\right| d \mu(S),
$$

where the integral is over all cuts $(S, \bar{S})$. Conversely, to every embedding $f: V \rightarrow$ $L_{1}$, we can associate a cut measure $\mu$ such that (1) holds. We will use this correspondence freely in what follows. When $V$ is a finite set (as it will be throughout), for $A \subseteq 2^{2^{V}}$, we define $\mu(A)=\sum_{S \in A} \mu(S)$.

Embeddings and Distortion If $\left(X, d_{X}\right),\left(Y, d_{Y}\right)$ are metric spaces, and $f: X \rightarrow Y$, then we write

$$
\|f\|_{\text {Lip }}=\sup _{x \neq y \in X} \frac{d_{Y}(f(x), f(y))}{d_{X}(x, y)} .
$$

If $f$ is injective, then the distortion of $f$ is defined by $\operatorname{dist}(f)=\|f\|_{\text {Lip }} \cdot\left\|f^{-1}\right\|_{\text {Lip }}$. A map with distortion $D$ will sometimes be referred to as D-bi-Lipschitz. If $d_{Y}(f(x), f(y)) \leq d_{X}(x, y)$ for every $x, y \in X$, we say that $f$ is non-expansive. For a metric space $X$, we use $c_{1}(X)$ to denote the least distortion required to embed $X$ into $L_{1}$. 


\section{Coarse Differentiation}

In the present section, we study the regularity of paths under bi-Lipschitz mappings into $L_{1}$. Our main tool is based on differentiation [15]. First, we need a discrete analog of bounded variation.

Definition 3.1 An ordered sequence $\left\{x_{1}, x_{2}, \ldots, x_{k}\right\} \subseteq X$ in a metric space $(X, d)$ is said to be $\epsilon$-efficient if

$$
d\left(x_{1}, x_{k}\right) \leq \sum_{i=1}^{k-1} d\left(x_{i}, x_{i+1}\right) \leq(1+\epsilon) d\left(x_{1}, x_{k}\right) .
$$

Of course the left inequality follows trivially from the triangle inequality.

Definition 3.2 A function $f: Y \rightarrow X$ between two metric spaces $(X, d)$ and $\left(Y, d^{\prime}\right)$ is said to be $\epsilon$-efficient on $P=\left\{y_{1}, y_{2}, \ldots, y_{k}\right\} \subseteq Y$ if the sequence $f(P)=$ $\left\{f\left(y_{1}\right), f\left(y_{2}\right), \ldots, f\left(y_{k}\right)\right\}$ is $\epsilon$-efficient in $X$.

For the sake of simplicity, we first present the coarse differentiation argument for a function on $[0,1]$. Let $f:[0,1] \rightarrow X$ be a non-expansive map into a metric space $(X, d)$. Let $C \in \mathbb{N}$ be given, and for each $k \in \mathbb{N}$, let $L_{k}=\left\{j C^{-k}\right\}_{j=0}^{C^{k}} \subseteq[0,1]$ be the set of level- $k$ points, and let $S_{k}=\left\{\left(j C^{-k},(j+1) C^{-k}\right): j \in\left\{0, \ldots, C^{k}-1\right\}\right\}$ be the set of level-k pairs.

For an interval $I=[a, b],\left.f\right|_{I}$ denotes the restriction of $f$ to the interval $I$. Now we say that $\left.f\right|_{I}$ is $\varepsilon$-efficient at granularity $C$ if

$$
\sum_{j=0}^{C-1} d\left(f\left(a+\frac{(b-a) j}{C}\right), f\left(a+\frac{(b-a)(j+1)}{C}\right)\right) \leq(1+\varepsilon) d(f(a), f(b)) .
$$

Further, we say that a function $f$ is $(\varepsilon, \delta)$-inefficient at level $k$ if

$$
\mid\left\{(a, b) \in S_{k}:\left.f\right|_{[a, b]} \text { is not } \varepsilon \text {-efficient at granularity } C\right\} \mid \geq \delta C^{k} .
$$

In other words, the probability that a randomly chosen level $k$ restriction $\left.f\right|_{[a, b]}$ is not $\varepsilon$-efficient is at least $\delta$. Otherwise, we say that $f$ is $(\varepsilon, \delta)$-efficient at level $k$. The main theorem of this section follows.

Theorem 3.3 (Coarse Differentiation) If a non-expansive map $f:[0,1] \rightarrow X$ is $(\varepsilon, \delta)$-inefficient at an $\alpha$-fraction of levels $k=1,2, \ldots, N$, then $\operatorname{dist}\left(\left.f\right|_{L_{N+1}}\right) \geq$ $\varepsilon \alpha \delta N$.

Proof Let $D=\operatorname{dist}\left(\left.f\right|_{L_{N+1}}\right)$, and let $1 \leq k_{1}<\cdots<k_{h} \leq N$ be the $h \geq \alpha N$ levels at which $f$ is $(\varepsilon, \delta)$-inefficient.

Let us consider the first level $k_{1}$. Let $S_{k_{1}}^{\prime} \subseteq S_{k_{1}}$ be a subset of size $\left|S_{k_{1}}^{\prime}\right| \geq \delta\left|S_{k_{1}}\right|$ for which

$$
\left.(a, b) \in S_{k_{1}}^{\prime} \Longrightarrow f\right|_{[a, b]} \quad \text { is not } \varepsilon \text {-efficient at granularity } C \text {. }
$$


For any such $(a, b) \in S_{k_{1}}^{\prime}$, we know that

$$
\begin{aligned}
\sum_{j=0}^{C-1} d\left(f\left(a+j C^{-k_{1}-1}\right), f\left(a+(j+1) C^{-k_{1}-1}\right)\right) & >(1+\varepsilon) d(f(a), f(b)) \\
& \geq d(f(a), f(b))+\varepsilon \frac{C^{-k_{1}}}{D}
\end{aligned}
$$

by the definition of (not being) $\varepsilon$-efficient and the fact that $d(f(a), f(b)) \geq \mid a-$ $b \mid / D$. For all segments $(a, b) \in S_{k_{1}} \backslash S_{k_{1}}^{\prime}$, the triangle inequality yields

$$
\sum_{j=0}^{C-1} d\left(f\left(a+j C^{-k_{1}-1}\right), f\left(a+(j+1) C^{-k_{1}-1}\right)\right) \geq d(f(a), f(b)) .
$$

By summing the above inequalities over all the segments in $S_{k_{1}}$, we get

$$
\sum_{(u, v) \in S_{k_{1}+1}} d(f(u), f(v)) \geq \sum_{(a, b) \in S_{k_{1}}} d(f(a), f(b))+\frac{\varepsilon \delta}{D} .
$$

Similarly, for each of the levels $k_{2}, \ldots, k_{h}$, we will pick up an excess term of $\varepsilon \delta / D$. We conclude that

$$
1 \geq \sum_{(u, v) \in S_{N+1}} d(f(u), f(v)) \geq \frac{\varepsilon \delta h}{D}
$$

where the LHS comes from the fact that $f$ is non-expansive. Simplifying achieves the desired conclusion.

\subsection{Differentiation for Families of Geodesics}

Let $G=(V, E)$ be an unweighted graph, and let $\mathcal{P}$ denote a family of geodesics (i.e., shortest-paths) in $G$. Furthermore, assume that every $\gamma \in \mathcal{P}$ has length $C^{r}$ for some $C$ and $r \in \mathbb{N}$. Let $f:\left(V, d_{G}\right) \rightarrow X$ be a non-expansive map into some metric space $(X, d)$.

For the sake of convenience, we will index the vertices along the paths using numbers from $[0,1]$. Specifically, we will refer to the $i$ th vertex along the path $\gamma \in \mathcal{P}$ by $\gamma\left(\frac{i}{C^{r}}\right)$. For indices $a, b$, we will use $\gamma[a, b]$ to denote the sub path starting at $\gamma(a)$ and ending at $\gamma(b)$. We will also use $\left.f\right|_{\gamma[a, b]}$ to denote the restriction of $f$ to the path $\gamma[a, b]$. As earlier, the function $\left.f\right|_{\gamma[a, b]}$ is said to be $\epsilon$-efficient at granularity $C$ if

$$
\begin{aligned}
& \sum_{j=0}^{C-1} d\left(f\left(\gamma\left(a+C^{-1}(b-a) j\right)\right), f\left(\gamma\left(a+C^{-1}(b-a)(j+1)\right)\right)\right) \\
& \quad \leq(1+\varepsilon) d(f(a), f(b)) .
\end{aligned}
$$


Let the sets $L_{k}$ and $S_{k}$ be defined as before. Thus a level- $k$ segment of a path $\gamma \in \mathcal{P}$ is $\gamma[a, b]$ for some $(a, b) \in S_{k}$. We say that $f$ is $(\epsilon, \delta)$ inefficient at level $k$ for the family of paths $\mathcal{P}$ if the following holds:

$$
\mid\left\{(a, b) \in S_{k}, \gamma \in \mathcal{P}:\left.f\right|_{\gamma[a, b]} \text { is not } \varepsilon \text {-efficient at granularity } C\right\}\left|\geq \delta C^{k}\right| \mathcal{P} \mid \text {. }
$$

A straightforward variation of the proof of Theorem 3.3 yields the following.

Theorem 3.4 If a non-expansive map $f: V \rightarrow X$ is $(\varepsilon, \delta)$-inefficient at an $\alpha$-fraction of levels $k=1,2, \ldots, N$, then $\operatorname{dist}(f) \geq \varepsilon \alpha \delta N$.

Proof Let $D=\operatorname{dist}(f)$, and let $1 \leq k_{1}<\cdots<k_{h} \leq N$ be the $h \geq \alpha N$ levels for which $f$ is $(\varepsilon, \delta)$-inefficient at level $k_{i}$.

Let us consider the first level $k_{1}$. Let $S_{k_{1}}^{\prime} \subseteq \mathcal{P} \times S_{k_{1}}$ be a subset of size $\left|S_{k_{1}}^{\prime}\right| \geq$ $\delta\left|S_{k_{1}}\right||\mathcal{P}|$ for which

$$
\left.(\gamma,(a, b)) \in S_{k_{1}}^{\prime} \Longrightarrow f\right|_{\gamma[a, b]} \text { is not } \varepsilon \text {-efficient at granularity } C \text {. }
$$

For any such $(\gamma,(a, b)) \in S_{k_{1}}^{\prime}$, we know that

$$
\begin{aligned}
& \sum_{j=0}^{C-1} d\left(f\left(\gamma\left(a+j C^{-k_{1}-1}\right)\right), f\left(\gamma\left(a+(j+1) C^{-k_{1}-1}\right)\right)\right) \\
& \quad>(1+\varepsilon) d(f(\gamma(a)), f(\gamma(b))) \\
& \quad \geq d(f(\gamma(a)), f(\gamma(b)))+\varepsilon \frac{C^{N-k_{1}}}{D}
\end{aligned}
$$

by the definition of (not being) $\varepsilon$-efficient and the fact that $d(f(\gamma(a)), f(\gamma(b))) \geq$ $C^{N}|a-b| / D$. In particular, summing both sides over all the segments $\gamma[a, b]$ over all paths $\gamma$ and segments $[a, b] \in S_{k_{1}}$ (and replacing the preceding inequality by the triangle inequality if $\left.(a, b) \notin S_{k_{1}}^{\prime}\right)$, we get

$$
\begin{aligned}
& \sum_{\gamma \in \mathcal{P}} \sum_{(u, v) \in S_{k_{1}+1}} d(f(\gamma(u)), f(\gamma(v))) \\
& \quad \geq \sum_{\gamma \in \mathcal{P}} \sum_{(a, b) \in S_{k_{1}}} d(f(\gamma(a)), f(\gamma(b)))+\frac{\varepsilon \delta C^{N}|\mathcal{P}|}{D} .
\end{aligned}
$$

Similarly, for each of the levels $k_{2}, \ldots, k_{h}$, we will pick up an excess term of $\varepsilon \delta C^{N}|\mathcal{P}| / D$. We conclude that

$$
C^{N}|\mathcal{P}| \geq \sum_{\gamma \in \mathcal{P}} \sum_{(u, v) \in S_{N+1}} d(f(\gamma(u)), f(\gamma(v))) \geq \frac{\varepsilon \delta h C^{N}|\mathcal{P}|}{D},
$$

and the desired conclusion follows. 


\subsection{Efficient $L_{1}$-valued Maps and Monotone Cuts}

Finally, we relate monotonicity of $L_{1}$-valued mappings to properties of their cut decompositions.

Definition 3.5 A sequence $P=\left\{x_{1}, x_{2}, \ldots, x_{k}\right\} \subseteq X$ is said to be monotone with respect to a cut $(S, \bar{S})$ (where $X=S \uplus \bar{S}$ ) if $S \cap P=\left\{x_{1}, x_{2}, \ldots, x_{i}\right\}$ or $\bar{S} \cap P=$ $\left\{x_{1}, x_{2}, \ldots, x_{i}\right\}$ for some $1 \leq i \leq k$.

If $\mu$ is a cut measure on a finite set $X$, we define the separation measure $\mu^{P}$ for a subset $P \subseteq X$ as follows: For every $S \subseteq X$, let

$$
\mu^{P}(S)= \begin{cases}\mu(S) & \text { if } P \nsubseteq S \wedge P \nsubseteq \bar{S} \\ 0 & \text { otherwise. }\end{cases}
$$

Lemma 3.6 Let $(X, d)$ be a finite metric space, and let $P=\left\{x_{1}, x_{2}, \ldots, x_{k}\right\} \subseteq X$ be a finite sequence. Given a mapping $f: X \rightarrow L_{1}$, let $\mu$ be the corresponding cut measure (see (1)). If $f$ is $\epsilon$-efficient on $P$, then

$$
\mu^{P}(\{S: P \text { is monotone with respect to }(S, \bar{S})\}) \geq(1-\epsilon)\left\|f\left(x_{1}\right)-f\left(x_{k}\right)\right\|_{1} \text {. }
$$

Proof If the sequence $P$ is not monotone with respect to a cut $(S, \bar{S})$, then

$$
\sum_{i=1}^{k-1}\left|\mathbf{1}_{S}\left(x_{i}\right)-\mathbf{1}_{S}\left(x_{i+1}\right)\right| \geq 2\left|\mathbf{1}_{S}\left(x_{1}\right)-\mathbf{1}_{S}\left(x_{k}\right)\right| .
$$

Now, let $\mathcal{E}=\{S: P$ is not monotone with respect to $(S, \bar{S})\}$, and for the sake of contradiction, assume that $\mu^{P}(\mathcal{E})>\varepsilon\left\|f\left(x_{1}\right)-f\left(x_{k}\right)\right\|_{1}$, then

$$
\begin{aligned}
& \sum_{i=1}^{k-1}\left\|f\left(x_{i}\right)-f\left(x_{i+1}\right)\right\|_{1} \\
& \quad=\sum_{i=1}^{k-1}\left[\int_{\mathcal{E}}\left|\mathbf{1}_{S}\left(x_{i}\right)-\mathbf{1}_{S}\left(x_{i+1}\right)\right| d \mu(S)+\int_{\overline{\mathcal{E}}}\left|\mathbf{1}_{S}\left(x_{i}\right)-\mathbf{1}_{S}\left(x_{i+1}\right)\right| d \mu(S)\right] \\
& \quad \geq 2 \int_{\mathcal{E}}\left|\mathbf{1}_{S}\left(x_{1}\right)-\mathbf{1}_{S}\left(x_{k}\right)\right| d \mu(S)+\int_{\overline{\mathcal{E}}}\left|\mathbf{1}_{S}\left(x_{1}\right)-\mathbf{1}_{S}\left(x_{k}\right)\right| d \mu(S) \\
& \quad=2 \mu^{P}(\mathcal{E})+\mu^{P}(\overline{\mathcal{E}}) \\
& >(1+\epsilon)\left\|f\left(x_{1}\right)-f\left(x_{k}\right)\right\|_{1},
\end{aligned}
$$

where we observe that $\left\|f\left(x_{1}\right)-f\left(x_{k}\right)\right\|_{1} \leq \mu^{P}(\mathcal{E})+\mu^{P}(\overline{\mathcal{E}})$. This is a contradiction, since $f$ is assumed to be $\epsilon$-efficient on $P$. 


\section{The Distortion Lower Bound}

Our lower bound examples are the recursively defined family of graphs $\left\{K_{2, n}^{\oslash k}\right\}_{k=1}^{\infty}$. We recall that the graphs $K_{2,2}^{\oslash k}$ are known as diamond graphs [17, 27].

Lemma 4.1 Let $G$ be an $s-t$ graph with a uniform length function, i.e., len $(e)=1$ for every $e \in E(G)$. Then for every $\epsilon, D>0$, there exists an integer $N=N(G, \varepsilon, D)$ such that the following holds: For any non-expansive map $f: G^{\oslash N} \rightarrow X$ with $\operatorname{dist}(f) \leq D$, there exists a copy $G^{\prime}$ of $G$ in $G^{\oslash N}$ such that $f$ is $\epsilon$-efficient on all $s-t$ geodesics in $G^{\prime}$.

Proof Let $C=d_{G}(s, t)$, and let $\mathcal{P}_{G}$ denote the family of $s-t$ geodesics in $G$. Fix $\delta=\frac{1}{\left|\mathcal{P}_{G}\right|}, \alpha=\frac{1}{2}$, and $N=\frac{4 D}{\epsilon \delta}$.

Let $\mathcal{P}$ denote the family of all $s-t$ geodesics in $G^{\oslash N}$. Each path in $\mathcal{P}$ is of length $C$ and consists of $C^{N}$ edges. From the choice of parameters, observe that $\epsilon \alpha \delta N>D$. Applying Theorem 3.4 to the family $\mathcal{P}$, any non-expansive map $f$ with $\operatorname{dist}(f) \leq D$ is $(\epsilon, \delta)$-efficient at an $\alpha=\frac{1}{2}$-fraction of levels $k=1,2, \ldots, N$. Specifically, there exists a level $k$ such that $f$ is $(\epsilon, \delta)$-efficient at level $k$.

For a uniformly random choice of path $\gamma \in \mathcal{P}$ and level- $k$ segment $(a, b)$ of $\gamma$, $\left.f\right|_{\gamma[a, b]}$ is not $\epsilon$-efficient at granularity $C$ with probability at most $\delta$. In case of the family $\mathcal{P}$, each of the level- $k$ segments is nothing more than an $s-t$ geodesic in a level- $k$ copy of $G$.

If, for at least one of the level- $k$ copies of $G, f$ is $\epsilon$-efficient on all the $s-t$ geodesics in that copy, the proof is complete. On the contrary, suppose that each level- $k$ copy has an $s-t$ geodesic on which $f$ is not $\epsilon$-efficient. Then in each level- $k$ copy at least a $\delta=\frac{1}{\mathcal{P}_{G}}$-fraction of the $s-t$ geodesics are $\epsilon$-inefficient. Observe that the level$k$ copies form a partition the set of all level- $k$ segments, with each level- $k$ segment being a $s-t$ geodesic in a unique level- $k$ copy. Therefore, if at least a $\delta$-fraction of the $s-t$ geodesics in each level- $k$ copy are $\epsilon$-inefficient, then at least a $\delta$-fraction of all level- $k$ segments are $\epsilon$-inefficient. This contradicts the fact that $f$ is $(\epsilon, \delta)$-efficient at level $k$.

Although we will not need it, the same type of argument proves the following generalization to weighted graphs $G$. The idea is that in $G^{\oslash N}$ for $N$ large enough, there exists a copy of a subdivision of $G$ with each edge finitely subdivided. Paying small distortion, we can approximate $G$ (up to uniform scaling) by this subdivided copy, where the latter is equipped with uniform edge lengths.

Lemma 4.2 Let $G$ be an $s-t$ graph with arbitrary nonnegative edge lengths len: $E(G) \rightarrow \mathbb{R}_{+}$. Then for every $\epsilon, D>0$, there exists an integer $N=N(G, \varepsilon, D$, len) such that the following holds: For any non-expansive map $f: G^{\oslash N} \rightarrow X$ with $\operatorname{dist}(f) \leq D$, there exists a copy $G^{\prime}$ of $G$ in $G^{\oslash N}$ such that $f$ is $\epsilon$-efficient on all $s-t$ geodesics in $G^{\prime}$.

In the graph $K_{2, n}$, we will refer to the $n$ vertices other than $s, t$ by $M=\left\{m_{i}\right\}_{i=1}^{n}$. 
Lemma 4.3 For $\epsilon<\frac{1}{2}$ and any function $f: V\left(K_{2, n}\right) \rightarrow L_{1}$ that is $\epsilon / n$-efficient with respect to each of the geodesics $s-m_{i}-t$ for $1 \leq i \leq n$, we have $\operatorname{dist}(f) \geq 2-\frac{2}{n}-2 \epsilon$.

Proof Let $\mu$ be the cut measure corresponding to $f$. By scaling, we may assume that

$$
\|f(s)-f(t)\|_{1}=\mu\left\{S: \mathbf{1}_{S}(s) \neq \mathbf{1}_{S}(t)\right\}=1 .
$$

Let $V=V\left(K_{2, n}\right)$. Without loss of generality, we assume that $\mu$ is supported on $2^{V} \backslash$ $\{\emptyset, V\}$. Let $\gamma_{i}$ be the geodesic $s-m_{i}-t$ for $i \in\{1,2, \ldots, n\}$. Define

$$
\mathcal{E}_{i}=\left\{S:(S, \bar{S}) \text { is not monotone with respect to } \gamma_{i}\right\}
$$

and $\mathcal{E}=\bigcup_{i=1}^{n} \mathcal{E}_{i}$. Since $f$ is $\epsilon / n$ efficient on every $\gamma_{i}$, by applying Lemma 3.6 we see that $\mu^{\gamma_{i}}\left(\mathcal{E}_{i}\right) \leq \epsilon / n$. For every $(S, \bar{S}) \in \mathcal{E}$, there exists $i$ such that $\mu^{\gamma_{i}}(S)=\mu(S)$. Hence by a union bound, we see that $\mu(\mathcal{E}) \leq \sum_{i=1}^{n} \mu^{\gamma_{i}}\left(\mathcal{E}_{i}\right) \leq \epsilon$.

Consider a cut $(S, \bar{S})$ that is monotone with respect to all the $\gamma_{i}$ geodesics and such that $\mu(S)>0$. Let us refer to these cuts as good cuts. By monotonicity and the fact that $S \notin\{0, V\}$, we know that $\left|\mathbf{1}_{S}(s)-\mathbf{1}_{S}(t)\right|=1$. Thus for a good cut $(S, \bar{S})$, we have

$$
\sum_{i, j \in[n]}\left|\mathbf{1}_{S}\left(m_{i}\right)-\mathbf{1}_{S}\left(m_{j}\right)\right|=2(|S|-1)(n-|S|-1) \leq \frac{n^{2}}{2} .
$$

It follows that

$$
\begin{aligned}
& \sum_{i, j \in[n]}\left\|f\left(m_{i}\right)-f\left(m_{j}\right)\right\|_{1} \\
& \quad=\int_{\mathcal{E}} \sum_{i, j \in[n]}\left|\mathbf{1}_{S}\left(m_{i}\right)-\mathbf{1}_{S}\left(m_{j}\right)\right| d \mu(S)+\int_{\overline{\mathcal{E}}_{i, j \in[n]}}\left|\mathbf{1}_{S}\left(m_{i}\right)-\mathbf{1}_{S}\left(m_{j}\right)\right| d \mu(S) \\
& \quad \leq \mu(\overline{\mathcal{E}}) \frac{n^{2}}{2}+\mu(\mathcal{E}) n^{2} \\
& \quad \leq(1-\epsilon) \frac{n^{2}}{2}+\epsilon n^{2} \\
& \quad=\frac{(1+\epsilon) n^{2}}{2}\|f(s)-f(t)\|_{1},
\end{aligned}
$$

where in the first inequality, we have used (2), and we recall that $\|f(s)-f(t)\|_{1}=1$.

Contrasting this with the fact that

$$
\sum_{i, j \in[n]} d_{K_{2, n}}\left(m_{i}, m_{j}\right)=n(n-1) d_{K_{2, n}}(s, t)
$$

yields

$$
\operatorname{dist}(f) \geq \frac{n(n-1)}{\frac{(1+\epsilon) n^{2}}{2}}=\frac{2}{1+\epsilon}\left(1-\frac{1}{n}\right) \geq 2-\frac{2}{n}-2 \epsilon
$$


Theorem 4.4 For any $n \geq 2, \lim _{k \rightarrow \infty} c_{1}\left(K_{2, n}^{\oslash k}\right) \geq 2-\frac{2}{n}$.

Proof For any $\epsilon^{\prime}>0$, let $N$ be the integer obtained by applying Lemma 4.1 to $K_{2, n}$ with $\epsilon=\epsilon^{\prime} / n, D=2$ and $G=K_{2, n}$. We will show that for any map $f: K_{2, n}^{\oslash N} \rightarrow L_{1}$, $\operatorname{dist}(f) \geq 2-\frac{2}{n}-2 \epsilon^{\prime}$. Without loss of generality, assume that $f$ is non-expansive. If $\operatorname{dist}(f) \leq 2$, then by Lemma 4.1 there exists a copy of $K_{2, n}$ in which $f$ is $\frac{\epsilon^{\prime}}{n}$-efficient on all the $s-t$ geodesics. Using Lemma 4.3, we see that on this copy of $K_{2, n}$ we get $\operatorname{dist}\left(\left.f\right|_{K_{2, n}}\right) \geq 2-\frac{2}{n}-2 \epsilon^{\prime}$. The result follows by taking $\epsilon^{\prime} \rightarrow 0$.

\section{Embeddings of $K_{2, n}^{\oslash k}$}

In this section, we show that for every fixed $n, \lim _{k \rightarrow \infty} c_{1}\left(K_{2, n}^{\oslash k}\right)<2$.

A Next-embedding Operator Let $T$ be a random variable ranging over subsets of $V\left(K_{2, n}^{\oslash k}\right)$, and let $S$ be a random variable ranging over subsets of $V\left(K_{2, n}\right)$. We define a random subset $P_{S}(T) \subseteq V\left(K_{2, n}^{\oslash k+1}\right)$ as follows. One moves from $K_{2, n}^{\oslash k}$ to $K_{2, n}^{\oslash k+1}$ by replacing every edge $(x, y) \in E\left(K_{2, n}^{\oslash k}\right)$ with a copy of $K_{2, n}$ which we will call $K_{2, n}^{(x, y)}$. For every edge $(x, y) \in K_{2, n}^{\oslash k}$, let $S^{(x, y)}$ be an independent copy of the cut $S$ (which ranges over subsets of $\left.V\left(K_{2, n}\right)\right)$. We form the cut $P_{S}(T) \subseteq V\left(K_{2, n}^{\oslash k+1}\right)$ as follows. If $(x, y) \in E\left(K_{2, n}^{\oslash k}\right)$, then for $v \in V\left(K_{2, n}^{(x, y)}\right)$, we put

$$
\mathbf{1}_{P_{S}(T)}(v)= \begin{cases}\mathbf{1}_{P_{S}(T)}\left(s\left(K_{2, n}^{(x, y)}\right)\right) & \text { if } \mathbf{1}_{S^{(x, y)}}(v)=\mathbf{1}_{S^{(x, y)}}\left(s\left(K_{2, n}^{(x, y)}\right)\right), \\ \mathbf{1}_{P_{S}(T)}\left(t\left(K_{2, n}^{(x, y)}\right)\right) & \text { otherwise. }\end{cases}
$$

We note that, strictly speaking, the operator $P_{S}$ depends on $n$ and $k$, but we allow these to be implicit parameters.

\subsection{Embeddings for Small $n$}

Consider the graph $K_{2, n}$ with vertex set $V=\{s, t\} \cup M$. An embedding in the style of [17] would define a random subset $S \subseteq V$ by selecting $M^{\prime} \subseteq M$ to contain each vertex from $M$ independently with probability $\frac{1}{2}$, and then setting $S=\{s\} \cup M^{\prime}$. The resulting embedding has distortion 2 since, for every pair $x, y \in M$, we have $\operatorname{Pr}\left[\mathbf{1}_{S}(x) \neq \mathbf{1}_{S}(y)\right]=\frac{1}{2}$. To do slightly better, we choose a uniformly random subset $M^{\prime} \subseteq M$ of size $\left\lfloor\frac{n}{2}\right\rfloor$ and set $S=\{s\} \cup M^{\prime}$ or $S=\{s\} \cup\left(M \backslash M^{\prime}\right)$, each with probability half. In this case, we have

$$
\operatorname{Pr}\left[\mathbf{1}_{S}(x) \neq \mathbf{1}_{S}(y)\right]=\frac{\left\lfloor\frac{n}{2}\right\rfloor \cdot\left\lfloor\frac{n+1}{2}\right\rfloor}{\left(\begin{array}{l}
n \\
2
\end{array}\right)}>\frac{1}{2},
$$

resulting in a distortion slightly better than 2 . A recursive application of these ideas results in $\lim _{k \rightarrow \infty} c_{1}\left(K_{2, n}^{\oslash k}\right)<2$ for every $n \geq 1$, though the calculation is complicated by the fact that the worst distortion is incurred for a pair $\{x, y\}$ with $x \in M(H)$ and $y \in M(G)$ where $H$ is a copy of $K_{2, n}^{\oslash k_{1}}$ and $G$ is a copy of $K_{2, n}^{\oslash k_{2}}$, and the 
relationship between $k_{1}$ and $k_{2}$ depends on $n$. (For instance, $c_{1}\left(K_{2,2}\right)=1$, while $\lim _{k \rightarrow \infty}\left(K_{2,2}^{\oslash k}\right)=\frac{4}{3}$.)

Theorem 5.1 For any $n, k \in \mathbb{N}$, we have $c_{1}\left(K_{2, n}^{\oslash k}\right) \leq 2-\frac{2}{2\left\lceil\frac{n}{2}\right\rceil+1}$.

Proof For simplicity, we prove the bound for $K_{2,2 n}^{\oslash k}$. A similar analysis holds for $K_{2,2 n+1}^{\oslash k}$. We define a random cut $S_{k} \subseteq V\left(K_{2,2 n}^{\oslash k}\right)$ inductively. For $k=1$, choose a uniformly random partition $M\left(K_{2,2 n}^{\oslash 1}\right)=M_{S} \cup M_{t}$ with $\left|M_{s}\right|=\left|M_{t}\right|=n$, and let $S_{1}=\left\{s\left(K_{2,2 n}^{\oslash 1}\right)\right\} \cup\left\{M_{s}\right\}$. The key fact which causes the distortion to be less than 2 is the following: For any $x, y \in M\left(K_{2,2 n}^{\oslash 1}\right)$, we have

$$
\operatorname{Pr}\left[\mathbf{1}_{S_{1}}(x) \neq \mathbf{1}_{S_{1}}(y)\right]=\frac{n^{2}}{\left(\begin{array}{c}
2 n \\
2
\end{array}\right)}=\frac{n}{2 n-1}>\frac{1}{2} .
$$

This follows because there are $\left(\begin{array}{c}2 n \\ 2\end{array}\right)$ pairs $\{x, y\} \in M\left(K_{2,2 n}^{\oslash 1}\right)$ and $n^{2}$ are separated by $S_{1}$.

Assume now that we have a random subset $S_{k} \subseteq V\left(K_{2,2 n}^{\oslash k}\right)$. We set $S_{k+1}=P_{S_{1}}\left(S_{k}\right)$ where $P_{S_{1}}$ is the operator defined above, which maps random subsets of $V\left(K_{2,2 n}^{\oslash k}\right)$ to random subsets of $V\left(K_{2,2 n}^{\oslash k+1}\right)$. In other words, $S_{k}=P_{S_{1}}^{k-1}\left(S_{1}\right)$.

Let $s_{0}=s\left(K_{2,2 n}^{\oslash k}\right)$ and $t_{0}=t\left(K_{2,2 n}^{\oslash k}\right)$. It is easy to see that the cut $S=S_{k}$ defined above is always monotone with respect to every $s_{0}-t_{0}$ shortest path in $K_{2,2 n}^{\oslash k}$, thus every such path has exactly one edge cut by $S_{k}$, and furthermore the cut edge is uniformly chosen from along the path, i.e., $\operatorname{Pr}\left[\mathbf{1}_{S}(x) \neq \mathbf{1}_{S}(y)\right]=2^{-k}$ for every $(x, y) \in E\left(K_{2,2 n}^{\oslash k}\right)$. In particular, it follows that if $u, v \in V\left(K_{2,2 n}^{\oslash k}\right)$ lie along the same simple $s_{0}-t_{0}$ path, then $\operatorname{Pr}\left[\mathbf{1}_{S}(u) \neq \mathbf{1}_{S}(v)\right]=2^{-k} d(u, v)$.

Now consider any $u, v \in V\left(K_{2,2 n}^{\oslash k}\right)$. Fix some shortest path $P$ from $u$ to $v$. By symmetry, we may assume that $P$ goes left (toward $s_{0}$ ) and then right (toward $t_{0}$ ). Let $s$ be the left-most point of $P$. In this case, $s=s(H)$ for some subgraph $H$ which is a copy of $K_{2,2 n}^{\oslash k^{\prime}}$ with $k^{\prime} \leq k$ and such that $u, v \in V(H)$; we let $t=t(H)$. We also have $d(u, v)=d(u, s)+d(s, v)$. Let $M=M(H)$, and fix $x, y \in M$ which lie along the $s-u-t$ and $s-v-t$ shortest-paths, respectively. Without loss of generality, we may assume that $d(s, v) \leq d(s, y)$. We need to consider two cases (see Fig. 2).

Case I: $d(u, s) \leq d(x, s)$.

For any pair $a, b \in V\left(K_{2,2 n}^{\oslash k}\right)$, we let $\mathcal{E}_{a, b}$ be the event $\left\{\mathbf{1}_{S}(a) \neq \mathbf{1}_{S}(b)\right\}$. In this case, we have $\operatorname{Pr}\left[\mathcal{E}_{u, v}\right]=\operatorname{Pr}\left[\mathcal{E}_{s, t}\right] \cdot \operatorname{Pr}\left[\mathcal{E}_{u, v} \mid \mathcal{E}_{s, t}\right]$. Since $s, t$ clearly lie on a shortest $s_{0}-t_{0}$ path, we have $\operatorname{Pr}\left[\mathcal{E}_{s, t}\right]=2^{-k} d(s, t)$. For any event $\mathcal{E}$, we let $\mu[\mathcal{E}]=\operatorname{Pr}\left[\mathcal{E} \mid \mathcal{E}_{s, t}\right]$. Now we calculate using (3),

$$
\begin{aligned}
\mu\left[\mathcal{E}_{u, v}\right] & \geq \mu\left[\mathcal{E}_{x, y}\right]\left(\mu\left[\mathcal{E}_{x, s}{ }^{\prime} \mathcal{E}_{x, y}\right] \mu\left[\mathcal{E}_{u, s}{ }^{\prime} \mathcal{E}_{x, s}, \mathcal{E}_{x, y}\right]+\mu\left[\mathcal{E}_{x, t}{ }^{\prime} \mathcal{E}_{x, y}\right] \mu\left[\mathcal{E}_{v, s}{ }^{\prime} \mathcal{E}_{x, t}, \mathcal{E}_{x, y}\right]\right) \\
& =\frac{n}{2 n-1}\left(\frac{1}{2} \cdot \frac{d(u, s)}{d(x, s)}+\frac{1}{2} \cdot \frac{d(v, s)}{d(y, s)}\right) \\
& =\frac{n}{2 n-1} \frac{d(u, v)}{d(s, t)} .
\end{aligned}
$$




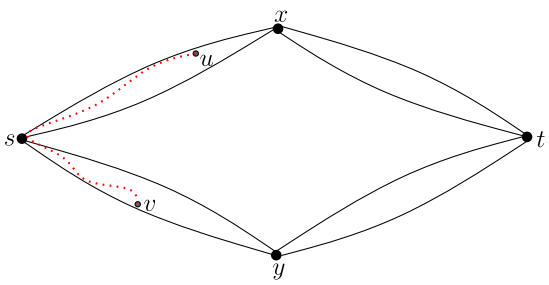

(a) Case I

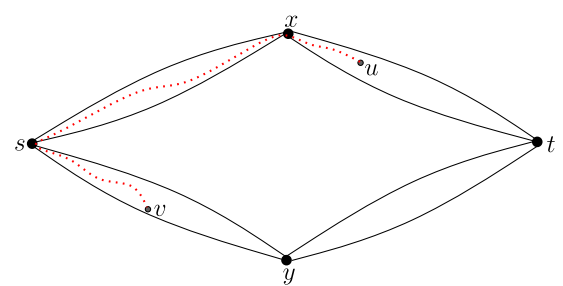

(b) Case II

Fig. 2 The two cases of Theorem 5.1

Hence in this case, $\operatorname{Pr}\left[\mathbf{1}_{S}(u) \neq \mathbf{1}_{S}(v)\right] \geq \frac{n}{2 n-1} \cdot 2^{-k} d(u, v)$.

Case II: $d(u, s) \geq d(x, s)$.

Here, we need to be more careful about bounding $\mu\left[\mathcal{E}_{u, v}\right]$. It will be helpful to introduce the notation $a \mapsto b$ to represent the event $\left\{\mathbf{1}_{S}(a)=\mathbf{1}_{S}(b)\right\}$. We have

$$
\begin{aligned}
\mu\left[\mathcal{E}_{u, v}\right]= & \mu[x \mapsto t, y \mapsto s]+\mu[x \mapsto t, y \mapsto t, v \mapsto s]+\mu[x \mapsto s, y \mapsto s, u \mapsto t] \\
& +\mu[x \mapsto s, y \mapsto t, u \mapsto t, v \mapsto s]+\mu[x \mapsto s, y \mapsto t, u \mapsto s, v \mapsto t] \\
= & \frac{1}{2} \frac{n}{2 n-1}+\frac{n-1}{2 n-1} \frac{d(v, y)+d(u, x)}{d(s, t)} \\
& +\frac{1}{2} \frac{n}{2 n-1}\left(\frac{d(u, x) d(v, y)+d(u, t) d(v, s)}{d(x, t) d(y, s)}\right) .
\end{aligned}
$$

If we set $A=\frac{d(v, s)}{d(s, t)}$ and $B=\frac{d(u, x)}{d(s, t)}$, then $\frac{d(u, v)}{d(s, t)}=\frac{1}{2}+A+B$, and simplifying the expression above, we have

$$
\mu\left[\mathcal{E}_{u, v}\right]=\frac{1}{2}+B+\frac{A}{2 n-1}-\frac{4 n}{2 n-1} A B .
$$

Since the shortest path from $u$ to $v$ goes through $s$ by assumption, we must have $A+B \leq \frac{1}{2}$. Thus we are interested in the minimum of $\mu\left[\mathcal{E}_{u, v}\right] /\left(\frac{1}{2}+A+B\right)$ subject to the constraint $A+B \leq \frac{1}{2}$. It is easy to see that the minimum is achieved at $A+B=\frac{1}{2}$, thus setting $B=\frac{1}{2}-A$, we are left to find

$$
\min _{0 \leq A \leq \frac{1}{2}}\left\{1-2 A+\frac{4 n A^{2}}{2 n-1}\right\}=\frac{2 n+1}{4 n} .
$$

(The minimum occurs at $A=\frac{1}{2}-\frac{1}{4 n}$.) So in this case, $\operatorname{Pr}\left[\mathbf{1}_{S}(u) \neq \mathbf{1}_{S}(v)\right] \geq$ $\frac{2 n+1}{4 n} 2^{-k} d(u, v)$.

Combining the above two cases, we conclude that the distribution $S=S_{k}$ induces an $L_{1}$ embedding of $K_{2,2 n}^{\oslash k}$ with distortion at $\operatorname{most} \max \left\{\frac{2 n-1}{n}, \frac{4 n}{2 n+1}\right\}=2-\frac{2}{2 n+1}$. 
A similar calculation yields

$$
c_{1}\left(K_{2,2 n+1}^{\oslash k}\right) \leq\left(\min _{0 \leq A \leq \frac{1}{2}}\left\{1-2 A+\frac{4(n+1) A^{2}}{2 n+1}\right\}\right)^{-1}=2-\frac{2}{2 n+3} .
$$

Acknowledgements We thank Alex Eskin for explaining coarse differentiation to us, Yuri Rabinovich for relating the distortion 2 conjecture, and Kostya and Yury Makayrchev for a number of fruitful discussions.

\section{References}

1. Andoni, A., Deza, M., Gupta, A., Indyk, P., Raskhodnikova, S.: Lower bounds for embedding of edit distance into normed spaces. In: Proceedings of the 14th Annual ACM-SIAM Symposium on Discrete Algorithms (2003)

2. Arora, S., Lee, J.R., Naor, A.: Euclidean distortion and the sparsest cut. J. Am. Math. Soc. 21(1), 1-21 (2008)

3. Arora, S., Rao, S., Vazirani, U.: Expander flows, geometric embeddings, and graph partitionings. In: 36th Annual Symposium on the Theory of Computing, pp. 222-231 (2004). J. ACM, to appear

4. Aumann, Y., Rabani, Y.: An $O(\log k)$ approximate min-cut max-flow theorem and approximation algorithm. SIAM J. Comput. 27(1), 291-301 (1998)

5. Benyamini, Y., Lindenstrauss, J.: Geometric Nonlinear Functional Analysis, vol. 1. American Mathematical Society Colloquium Publications, vol. 48. American Mathematical Society, Providence (2000)

6. Bourgain, J.: On Lipschitz embedding of finite metric spaces in Hilbert space. Israel J. Math. 52(1-2), 46-52 (1985)

7. Brinkman, B., Karagiozova, A., Lee, J.R.: Vertex cuts, random walks, and dimension reduction in series-parallel graphs. In: 39th Annual Symposium on the Theory of Computing, pp. 621-630 (2007)

8. Chakrabarti, A., Jaffe, A., Lee, J.R., Vincent, J.: Embeddings of topological graphs: Lossy invariants, linearization, and 2-sums. In: 49th Annual Symposium on Foundations of Computer Science, pp. 761770 (2008)

9. Cheeger, J.: Differentiability of Lipschitz functions on metric measure spaces. Geom. Funct. Anal. 9(3), 428-517 (1999)

10. Cheeger, J., Kleiner, B.: Differentiating maps into $l^{1}$ and the geometry of bv functions. Preprint: math/0611954v3 (2006)

11. Cheeger, J., Kleiner, B.: Generalized differential and bi-Lipschitz nonembedding in $L^{1}$. C. R. Math. Acad. Sci. Paris 343(5), 297-301 (2006)

12. Chekuri, C., Gupta, A., Newman, I., Rabinovich, Y., Sinclair, A.: Embedding $k$-outerplanar graphs into $l_{1}$. SIAM J. Discrete Math. 20(1), 119-136 (2006)

13. Deza, M.M., Laurent, M.: Geometry of Cuts and Metrics. Algorithms and Combinatorics, vol. 15. Springer, Berlin (1997)

14. Diestel, R.: Graph Theory, 3rd edn. Graduate Texts in Mathematics, vol. 173. Springer, Berlin (2005)

15. Eskin, A., Fisher, D., Whyte, K.: Quasi-isometries and rigidity of solvable groups. Preprint (2006)

16. Franchi, B., Serapioni, R., Serra Cassano, F.: Rectifiability and perimeter in the Heisenberg group. Math. Ann. 321(3), 479-531 (2001)

17. Gupta, A., Newman, I., Rabinovich, Y., Sinclair, A.: Cuts, trees and $l_{1}$-embeddings of graphs. Combinatorica 24(2), 233-269 (2004)

18. Heinonen, J.: Lectures on Analysis on Metric Spaces. Universitext. Springer, New York (2001)

19. Indyk, P.: Algorithmic applications of low-distortion geometric embeddings. In: 42nd Annual Symposium on Foundations of Computer Science, pp. 10-33. IEEE Computer Society, Los Alamitos (2001)

20. Khot, S., Naor, A.: Nonembeddability theorems via Fourier analysis. Math. Ann. 334(4), 821-852 (2006)

21. Khot, S., Vishnoi, N.: The unique games conjecture, integrability gap for cut problems and embeddability of negative type metrics into $\ell_{1}$. In: 46th Annual Symposium on Foundations of Computer Science, pp. 53-62. IEEE Computer Society, Los Alamitos (2005) 
22. Lee, J.R., Naor, A.: $l_{p}$ metrics on the Heisenberg group and the Goemans-Linial conjecture. In: 47th Annual Symposium on Foundations of Computer Science. IEEE Computer Society, Los Alamitos (2006)

23. Linial, N.: Finite metric-spaces-combinatorics, geometry and algorithms. In: Proceedings of the International Congress of Mathematicians, vol. III, Beijing, 2002, pp. 573-586. Higher Education Press, Beijing (2002)

24. Linial, N., London, E., Rabinovich, Y.: The geometry of graphs and some of its algorithmic applications. Combinatorica 15(2), 215-245 (1995)

25. Matoušek, J.: Open problems on low-distortion embeddings of finite metric spaces. http://kam.mff. cuni.cz/ matousek/metrop.ps

26. Matoušek, J.: Lectures on Discrete Geometry. Graduate Texts in Mathematics, vol. 212. Springer, New York (2002)

27. Newman, I., Rabinovich, Y.: A lower bound on the distortion of embedding planar metrics into Euclidean space. Discrete Comput. Geom. 29(1), 77-81 (2003)

28. Okamura, H., Seymour, P.D.: Multicommodity flows in planar graphs. J. Comb. Theory Ser. B 31(1), 75-81 (1981)

29. Pansu, P.: Métriques de Carnot-Carathéodory et quasiisométries des espaces symétriques de rang un. Ann. Math. (2) 129(1), 1-60 (1989) 